\title{
Advanced Platform for 3D Visualization, Reconstruction, and Segmentation with Electron Tomography
}

\author{
Yi Jiang ${ }^{1}$, Marcus D. Hanwell ${ }^{2}$, Elliot Padgett ${ }^{3}$, Shawn Waldon ${ }^{2}$, David A. Muller ${ }^{3,4}$, Robert Hovden ${ }^{3}$ \\ 1. Department of Physics, Cornell University, Ithaca, NY, USA \\ 2. Kitware, Inc., Clifton Park, NY, USA \\ 3. School of Applied \& Engineering Physics, Cornell University, Ithaca NY, USA \\ 4. Kavli Institute at Cornell for Nanoscale Science, Ithaca, New York
}

Three-dimensional (3D) characterization of materials at the nano- and meso-scale has become possible with electron tomography (ET) [1-2]. The typical workflow consists of raw data collection with a (scanning) transmission electron microscope, tilt series processing/alignment, tomographic reconstruction, post processing, visualization and analysis. Each step often requires distinct software and has a significant impact to final results. This makes the workflow inefficient and impedes sharing of published or working data among researchers. To address this problem, we have developed an open and extendable platform, tomviz (www.tomviz.org), which integrates the entire ET workflow by providing essential tools from basic image filters to 3D animations (Figure 1).

For raw data (i.e. tilt series), tomviz provides both manual and automatic tools for translation and tilt axis alignment, background subtraction, and noise reduction. The platform includes a broad collection of reconstruction methods, from weighted back projection to more advanced optimization-based techniques such as the total-variation minimization method [3]. Moreover, for algorithms that involve additional optimization parameters, tomviz provides several image analysis methods that facilitate automatic parameter selection as well as reconstruction validation.

Combining the popular Insight Toolkit (ITK) and the Visualization Toolkit (VTK) [4], tomviz can perform fast and interactive 3D visualization and segmentation (Figure 2). The platform offers a variety of GPU-accelerated rendering modes such as shaded contours or volumetric projections, which can be used in combination with more than 100 color maps. Multiple objects can be rotated and transformed at the same time, enabling comparison of time resolved tomographic reconstructions and visualization of hyper-dimensional data such as 3D chemical mapping.

tomviz is cross-platform, open source, with installers available for Windows and Mac OS X. It is written in $\mathrm{C}++$ and Python (with built-in Numpy, Scipy, and wrapped ITK/VTK) and has flexible plug-in capabilities for users to implement their own algorithms and applications. With tomviz, the full pipeline of data processing steps can be presented, saved, and restored, enabling fully reproducible results for inter-laboratory comparison. Users can also conveniently access and modify the source code of each data transform. Furthermore, tomviz includes several sample ET datasets and reconstructions, which makes the software a valuable resource for learning electron tomography and testing methods. [5]

\section{References:}

[1] De Rosier, D. and Klug, A. Nature 217, 130-134 (1968)

[2] Midgley, P.A. et al., Chemical Communications, 10, 907-908 (2001)

[3] E. Sidky et al, Physics in medicine and biology 53 (2008), p.4777-4807.

[4] M.D. Hanwell et al, SoftwareX 1-2, 9-12 (2015) 
[5] tomviz is supported from DOE Office of Science contract DE-SC0011385.

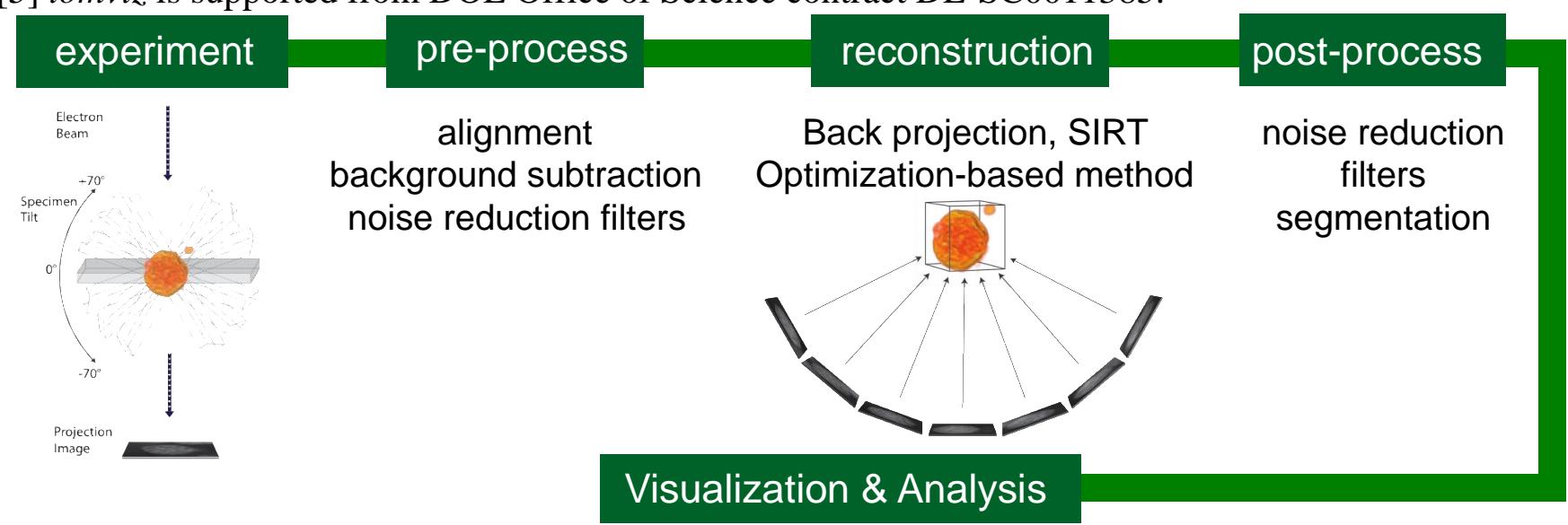

Figure 1. Electron tomography workflow in tomviz. The raw data first undergoes a pre-processing step, including alignment, background subtraction, etc. Next, the 3D model of specimen can be obtained by various reconstruction algorithms such as back projection and optimization-based methods. Users can further process and segment the reconstruction for visulization and statistical analysis.

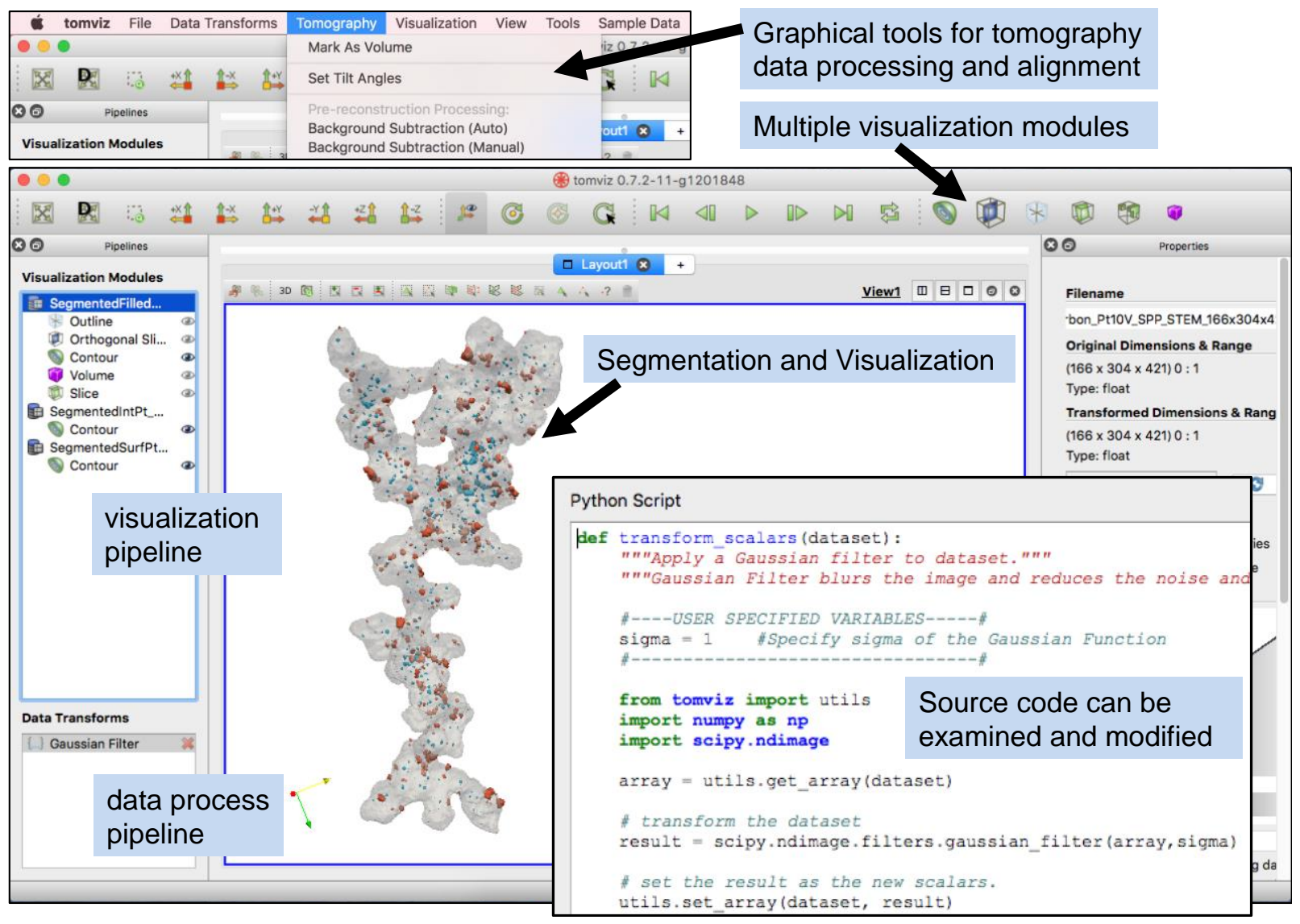

Figure 2. tomviz provides intuitive graphical interface for tomographic reconstruction, segmentation and visualization. Source-code of each data transform can be modified and the entire pipeline can be saved, restored, and shared. 